NASA-TM-112145

\title{
Potential flow downstream of the heliospheric terminal shock: A non-spherical shock
}

\author{
Steven Nerney ${ }^{1}$ and S. T. Suess \\ Space Science Lab/ES82, NASA Marshall Space Flight Center, Huntsville, Alabama
}

\begin{abstract}
We have solved for the potential flow downstream of the terminal shock of the solar wind in the limit of small departures from a spherical shock due to a latitudinal ram pressure variation in the supersonic solar wind. The solution connects anisotropic streamlines at the shock to uniform streamlines down the heliotail because we use a non-slip boundary condition on the heliopause at large radii. The rotational velocity about the heliotail in the near-field solution decays as the fourth power of distance from the shock. The polar divergence of the streamlines will have consequences for the previously discussed magnetic pressure ridge that may build-up just inside the heliopause.
\end{abstract}

\section{Introduction}

We have previously shown the conditions under which potential flow occurs beyond the terminal shock of the solar wind. Topologically, potential flow represents well many of the global properties which are universal to all heliospheric stagnation point flow topologies [Suess, 1990]. The flow is self-consistently incompressible to about $15 \%$ and irrotational to $10 \%$ [Suess and Nerney, 1990,1991 (SN1, SN2)]. The latter is true when the non-slip condition is used on the heliopause at large radii. Because it is likely that reconnection occurs near the stagnation point between the heliosheath and interstellar magnetic fields, we believe that the heliopause is best represented as a non-slip interface [Suess and Nerney, 1993]. This reduces the solar wind Mach number, $\mathrm{M}$, down the heliotail compared with free-slip numerical models. Then the effect of compressibility in this region is reduced by $M^{2}$. Recently, a numerical simulation for an external Mach number of $\mathrm{M}=0.8$ has shown that the shock is nearly spherical and Sun-centered even in that extreme case [Steinolfson, 1994]. That calculation showed that the nose-to-tail density in the heliosheath changes by a factor of two; our use of a non-slip heliopause greatly reduces this effect.

We do not consider the case of external supersonic flow because we believe that the interstellar wind is

\footnotetext{
${ }^{1}$ Ohio University, Lancaster, OH
}

Copyright 1995 by the American Geophysical Union.

Paper number 95GL01658

0094-8534/95/95GL-01658\$03.00 likely to be sub-Alfvenic and information will be able to propagate upstream, removing the necessity for a bow shock (also see the discussion in Nerney et al., 1995).

The merging of the shocked, subsonic solar wind with the $25 \mathrm{~km} / \mathrm{s}$ interstellar wind was calculated in our potential models by including the effects of a finite radius $\left(R_{s}\right)$ terminal shock. This effect entered the solution for the streamlines through a parameter $\epsilon$ which is a measure of the ratio of the interstellar ram pressure to that of the pressure in the shocked solar wind just beyond the terminal shock. Vanishingly small values of $\epsilon$ reproduce Parker's [1963] model for a weak interstellar medium with a large turning radius for the solar wind. Because of this, the shock radius is small compared to the transverse dimension of the heliosphere, and the shock appears to have a negligible radius. The numerical solutions were plotted for variable $\epsilon$, and the distance from the stagnation point to the terminal shock was no larger than $R_{s}$ for probable heliospheric parameters.

It is our purpose now to show the nature of the change in these results when a heliocentric latitudinal ram pressure variation is imposed on the supersonic wind upstream of the shock. This requires a non-spherical shape for the shock due to, for instance, high-speed streams emanating from the poles of the sun, and changes the character of the solutions near the shock. Observations of a latitudinal variation in the interplanetary Lyman $\alpha$ distribution [ Kumar and Broadfoot, 1979; Lallement et al., 1985] have been interpreted as indicating a 30$50 \%$ decrease in the solar wind mass flux from solar equator to pole during 1973-1977. This would not lead to a large equator-to-pole gas pressure variation on the shock for our model. The Bernoulli equation analysis in SN1 (p.6409) shows that the pressure just downstream of the shock only depends on the pressure at large distances down the tail on each streamline to about $6 \%$. Because we have no velocity shear across the tail at large radii, the pressure is the same on all streamlines at large $r$ and equal to that in the interstellar medium, $P_{i \infty}$. Because we have linearized the solutions in the departure from sphericity, we are limited to the low end of the equator-to pole mass flux decrease.

The mapping from heliocentric spherical polar coordinates to heliocentric heliotail coordinates (cylindrical) introduces an azimuthal dependence of the streamlines about the heliotail. There is also rotational flow about the heliotail which decays as the fourth power of distance from the shock. A further asymmetry is introduced because the footpoints for each streamline begin at different distances from the sun. 


\section{Mathematical Formalism}

We solve the incompressible, irrotational (potential flow) equation for fluid flow:

$$
\nabla^{2} \Phi=0 .
$$

The velocity is derived from $-\nabla \Phi$ subject to the boundary conditions:

$$
\begin{gathered}
\left.\hat{e}_{r} \cdot \vec{v}(\theta, \phi)\right|_{R_{s}}=v_{s}(\theta, \phi) \\
\lim _{z \rightarrow \infty} \vec{v}=v_{i \infty} \hat{e}_{z}
\end{gathered}
$$

where $R_{s}$ is the distance to the terminal shock, $v_{s}$ is the solar wind flow velocity downstream of the shock, $\phi$ is the azimuthal angle about the heliotail, and $\theta$ is the polar angle with respect to the $\mathrm{z}$-axis, the direction of the interstellar wind. We impose the following asymptotic flow pattern on the supersonic solar wind:

$$
v_{s}=v_{s 0}\left(1-\delta \sin ^{2} \theta_{S}\right)
$$

where $\theta_{S}$ is the solar polar angle and $\delta \leq .2$, and $v_{s 0}$ is the solar wind velocity downstream of the shock along the solar rotation axis. Rotating into heliotail, heliocentric spherical polar coordinates:

$$
v_{s}=v_{s 0}\left[1-\delta\left(\cos ^{2} \theta+\sin ^{2} \theta \cos ^{2} \phi\right)\right] .
$$

The rotation of coordinate systems introduces a $\cos ^{2} \phi$ dependence on azimuth about the heliotail. Equivalently,

$$
v_{s}=v_{s 0}\left[1-\frac{\delta}{6}\left(4 P_{0}+2 P_{2}+P_{2}^{2} \cos 2 \phi\right)\right]
$$

where $P_{l}$ and $P_{l}^{m}$ are the Legendre polynomials and the associated Legendre polynomials, respectively.

Now, $\Phi$ may be written as:

$$
\Phi=\sum_{m, n}\left[A_{m, n}\left(\frac{r}{R_{s 0}}\right)^{n}+C_{m, n}\left(\frac{R_{s 0}}{r}\right)^{n+1}\right] \times
$$

and $R_{s 0}$ is the value of $R_{s}$ along the solar rotation axis. The $A_{m, n}$ are determined by requiring that the velocity of the shocked wind approach that of the interstellar medium at large $\mathbf{r}$, eq.(2b). As in $\mathrm{SN} \mathbf{I}$

where

$$
A_{m, n}=0 \quad \text { except } \quad \Lambda_{0,1}=-\mathrm{v}_{\mathrm{i} \infty} \mathrm{R}_{\mathrm{s} 0}
$$

$$
R_{s}=\frac{R_{s 0} v_{s}(\theta, \phi)}{v_{s 0}}=R_{s 0} V
$$

which is derived by setting the ram pressure of the solar wind equal to the net pressure in the local interstellar medium (LISM). Eq. (6) serves to define $V$ and gives the angular variation for the distance to the termination shock.

Taking the radial derivative of equation (4), including only terms present in $v_{s}$, and evaluating at $R_{s}$, we find:

$$
\begin{array}{r}
V=\epsilon^{2 / 3} P_{1}+\frac{1}{R_{s 0} v_{s 0} V^{2}}\left[C_{0,0}+\frac{2 C_{0,1} P_{1}}{V}\right. \\
\left.+\frac{3}{V^{2}}\left(C_{0,2} P_{2}^{0}+C_{2,2} P_{2}^{2} \cos 2 \phi\right)\right]
\end{array}
$$

so that the angular variables occur in both the numerators and denominators of equation (7), unlike the spherical shock solutions. We have defined $\epsilon$ as in SN2:

$$
\epsilon=\left[\frac{v_{i \infty}}{v_{s 0}}\right]^{3 / 2} .
$$

Now equating equations (3c) and (7) and linearizing the various powers of $1 / \mathrm{V}$ using binomial expansions, allows us to solve for the coefficients of the spherical harmonics so that $\Phi$ may be written as:

$$
\begin{gathered}
\frac{\Phi}{v_{s 0} R_{s 0}}=-\epsilon^{2 / 3} P_{1}\left[\frac{r}{R_{s 0}}+\frac{R_{s 0}^{2}}{2 r^{2}}\left(1-\frac{12}{5} \delta\right)\right] \\
+\frac{R_{s 0}}{r}(1-2 \delta)-\frac{R_{s 0}^{3}}{3 r^{3}} \delta\left(P_{2}+.5 P_{2}^{2} \cos 2 \phi\right)
\end{gathered}
$$

where

$$
\begin{gathered}
r^{2}=\varpi^{2}+z^{2} \\
P_{2}=\frac{1}{3}\left(3 \cos ^{2} \theta-1\right)
\end{gathered}
$$

and

$$
P_{2}^{2}=3 \sin ^{2} \theta
$$

The velocities are derived from the derivatives of $\Phi$.

$$
\begin{gathered}
v_{r}=-\frac{\partial \Phi}{\partial r} \\
\frac{v_{r}}{v_{s 0}}=\epsilon^{2 / 3}\left[1-\frac{R_{s 0}^{3}}{r^{3}}\left(1-\frac{12 \delta}{5}\right)\right] \cos \theta+\frac{R_{s 0}^{2}}{r^{2}}(1-2 \delta) \\
-\frac{.5 R_{s 0}^{4}}{r^{4}} \delta\left(3 \cos ^{2} \theta-1+3 \sin ^{2} \theta \cos 2 \phi\right) \\
v_{\theta}=-\frac{1}{r} \frac{\partial \Phi}{\partial \theta} \\
\frac{v_{\theta}}{v_{s 0}}=-\epsilon^{2 / 3} \sin \theta\left[1+.5 \frac{R_{s 0}^{3}}{r^{3}}\left(1-\frac{12 \delta}{5}\right)\right] \\
-.5 \delta \frac{R_{s}^{4}}{r^{4}} \sin 2 \theta(1-\cos 2 \phi) \\
v_{\phi}=-\frac{1}{\varpi} \frac{\partial \Phi}{\partial \phi} \\
\frac{v_{\phi}}{v_{s 0}}=-\delta \frac{R_{s 0}^{4}}{r^{4}} \sin \theta \sin 2 \phi
\end{gathered}
$$

In passing, we note, as expected, that there are no order $\epsilon$ effects in $v_{\phi}$.

We will now switch to cylindrical coordinates aligned with the heliotail and convert the derivatives in (11) to:

$$
\begin{gathered}
\frac{v_{\varpi}}{v_{s 0}}=\frac{R_{s 0}^{2} \varpi}{r^{3}}\left[1-\delta F_{1}-1.5 \epsilon^{2 / 3} \frac{z R_{s 0}}{r^{2}}\left(1-\frac{12 \delta}{5}\right)\right]_{(12 a)} \\
F_{1}=2+\frac{R_{s 0}^{2}}{2 r^{4}}\left[4 z^{2}-\varpi^{2}+\left(3 \varpi^{2}-2 z^{2}\right) \cos 2 \phi\right] \quad(12 b) \\
\frac{v_{z}}{v_{s 0}}=\frac{z R_{s 0}^{2}}{r^{3}}\left(1-\delta F_{2}\right) \\
-\epsilon^{2 / 3}\left[\frac{R_{s 0}^{3}}{2 r^{5}}\left(2 z^{2}-\varpi^{2}\right)\left(1-\frac{12}{5} \delta\right)-1\right] \quad(12 c) \\
F_{2}=2-.5 \frac{R_{s 0}^{2}}{r^{2}}+1.5 \frac{z^{2} R_{s 0}^{2}}{r^{4}}+\frac{\varpi^{2} R_{s 0}^{2}}{r^{4}}(2.5 \cos 2 \phi-1)
\end{gathered}
$$




$$
\frac{v_{\phi}}{v_{s 0}}=-\delta \frac{R_{s 0}^{4} \varpi}{r^{5}} \sin 2 \phi
$$

For economy of notation we have written $r^{2}$ for $\varpi^{2}+z^{2}$. The streamlines are defined by

$$
\frac{d z}{v_{z}}=\frac{d \varpi}{v_{\varpi}}=\frac{\varpi d \phi}{v_{\phi}}
$$

Using the nondimensional variables from $\mathrm{SN} 1$

$$
\eta=\frac{\varpi \epsilon^{1 / 3}}{R_{s 0}} \quad ; \quad \zeta=\frac{z \epsilon^{1 / 3}}{R_{s 0}}
$$

(note that $\epsilon^{1 / 3} / R_{s 0}$ remains finite as $\epsilon \rightarrow 0$ ) the streamline equations can be written as:

$$
\begin{gathered}
\frac{d \zeta}{\zeta\left(1-\delta F_{2}\right)+r_{n d}^{3}-\epsilon \frac{2 \zeta^{2}-\eta^{2}}{2 r_{n d}^{2}}\left(1-\frac{12}{5} \delta\right)}= \\
\frac{d \eta}{\eta\left[1-\delta F_{1}-\frac{1.5 \epsilon \zeta}{r_{n d}^{2}}\left(1-\frac{12 \delta}{5}\right)\right]}=-\frac{d \phi}{\frac{\delta \epsilon^{2 / 3} \sin 2 \phi}{r_{n d}^{2}}}
\end{gathered}
$$

where

$$
r_{n d}^{2}=\eta^{2}+\zeta^{2}
$$

and $F_{1}, F_{2}$ are rewritten as

$$
\begin{gathered}
F_{1}=2+\frac{\epsilon^{2 / 3}}{2 r_{n d}^{4}}\left[4 \zeta^{2}-\eta^{2}+\left(3 \eta^{2}-2 \zeta^{2}\right) \cos 2 \phi\right] \\
F_{2}=2-\frac{\epsilon^{2 / 3}}{r_{n d}^{2}}\left[.5-\frac{1.5 \zeta^{2}}{r_{n d}^{2}}-\frac{\eta^{2}}{r_{n d}^{2}}(2.5 \cos 2 \phi-1)\right]
\end{gathered}
$$

When $\eta=0$ and $d \eta / d \zeta$ is infinite, $v_{z}$ must be zero. Setting the left denominator of (15) to zero then determines the non-dimensional distance to the stagnation point:

$$
\zeta_{c}\left(1-\delta F_{2}\right)+\left(\zeta_{c}^{2}\right)^{3 / 2}-\epsilon(1-2.4 \delta)=0
$$

\section{Numerical Analysis}

Figure 1 shows the streamlines for a $20 \%$ deviation from a spherical shock both in the meridional plane (solid lines; $\phi=90^{\circ}$, which includes the solar rotation axis) and in the equatorial plane (dashed lines; $\phi=0^{\circ}$ ). The equatorial streamlines map back to the inner circle, a cross-section of the terminal shock, while the meridional streamlines map back to a cross-section of a prolate spheroid and show the $20 \%$ bulge over the poles. The positions of the heliopause for the polar and equatorial streamlines are indicated on the right edge of the graph, showing that the bulge in the shock over the poles pushes the heliopause out in the meridional plane compared to the equatorial plane. The streamlines are nearly radial on the outside of the shock for $\phi=90^{\circ}$, but there is elevated non-radial flow on the shock for $\phi=0^{\circ}$ and $\epsilon=.125$ (an appropriate value for the heliosphere). We choose to superimpose the graphs of the streamlines for $\phi=0^{\circ}, 90^{\circ}$ because these are the two angles about the heliotail for which $v_{\phi}$ is zero (see eq. (11d)). The following plots indicate that the problem is inherently three-dimensional for any other value of $\phi$.

Figures $2 a, b$, and $c$ show three views of streamlines that begin on the terminal shock at $\phi=60^{\circ}$, followed by
NON-SPHERICAL SHOCK $20 \%$ DEVIATION

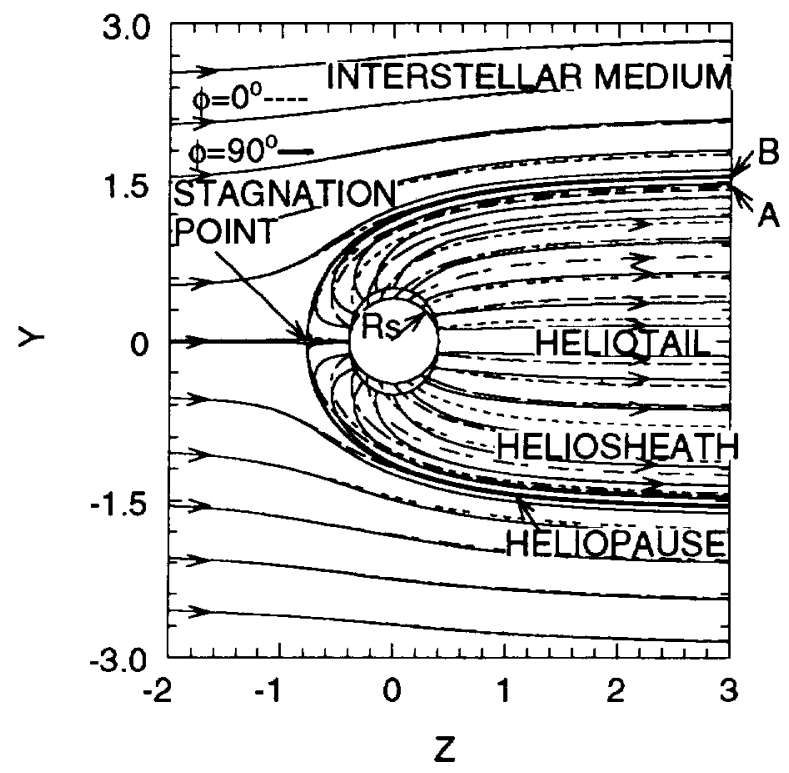

Figure 1. Figure 1. A plot of streamlines for the equatorial plane $\left(\phi=0^{\circ}\right.$; dashed lines and circular shock) and the meridional plane $\left(\phi=90^{\circ}\right.$; solid lines and oval shock for a $20 \%$ elongation over the poles). We use $\epsilon=.125$ - an appropriate choice for the heliosphere. Arrows on the right-side of the figure (A for $\phi=0^{\circ}$, B for $90^{\circ}$ ) point to the respective heliopauses. Streamlines are fully three-dimensional for other $\phi$.

fig $2 \mathrm{~d}$ which superimposes both $\phi=30^{\circ}$ (grey curves) and $\phi=60^{\circ}$ (black curves). The effects of $v_{\phi}$ are seen in the near-field solutions for the potential flow but decay asymptotically as $r^{-4}$ (see eq. (11d)).

The potential flow solutions connect the axisymmetric boundary condition at infinity (all streamlines blend into the assumed uniform interstellar wind) with the anisotropic boundary condition on the terminal shock (high-speed streams at the poles) so that the streamlines must diverge away from the poles more than at the equator. Another way to describe this effect is to remember that the solution to Laplace's equation minimizes the total kinetic energy per gram integrated over any volume bounded by streamlines. This requires a divergence of the flow away from the poles where the kinetic energy per gram on the shock is high.

\section{Summary and Conclusions}

We have solved a potential flow model for the flow down stream from a non-spherical terminal shock. The equations were linearized for small departures from sphericity and the streamline equations were numerically integrated to show the three-dimensional flow effects. The anisotropic streamlines on the terminal shock diverge away from the polar regions, while in the distant heliotail they smoothly take on the characteristics of the assumed uniform streamlines in the interstellar medium. 'This would have consequences for the previously reported ridge of magnetic flux that may buildup just inside of the heliopause [Nerney, Suess, and Schmahl, 1993]. The divergence of the flow away from the poles will initially open magnetic flux tubes, reducing the magnetic flux in the ridge. We have also 


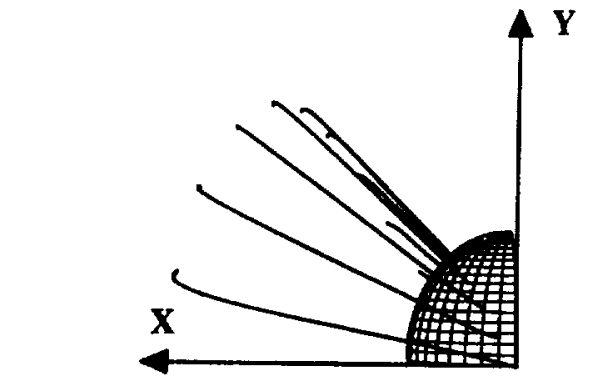

(a)

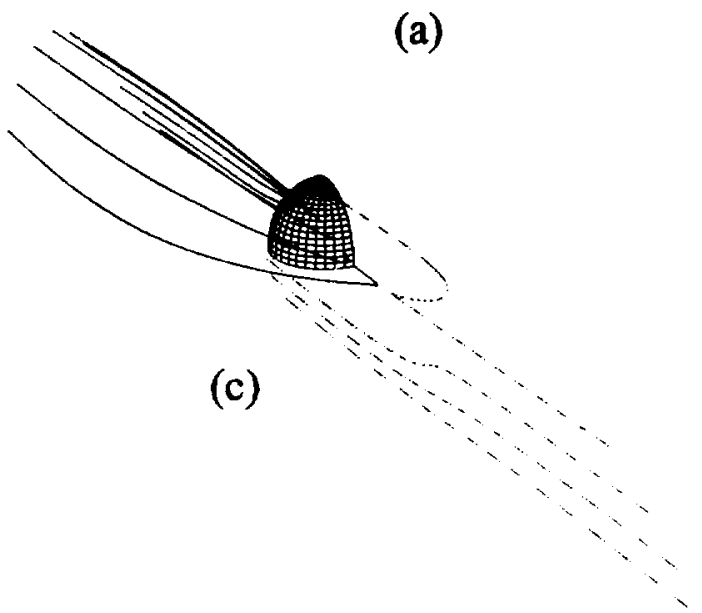

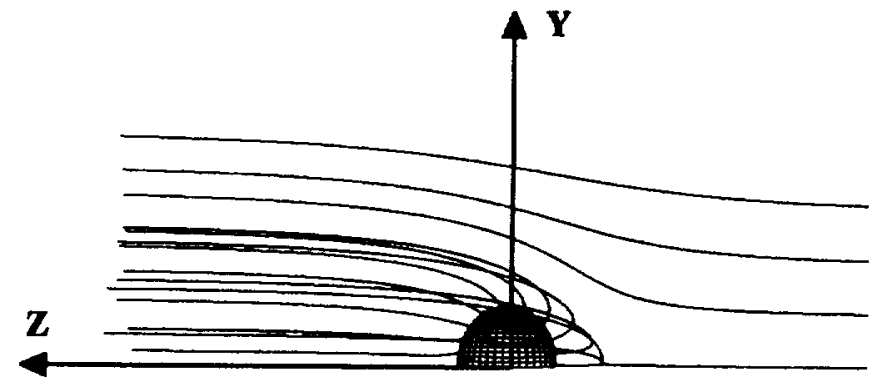

(b)

Figure 2. Figure 2. Panels (a), (b), and (c) show different views of the streamlines that begin on the terminal shock at $\phi=60^{\circ}$, while (d) shows a superposition of $\phi=30^{\circ}$ streamlines (grey curves) and $\phi=60^{\circ}$ streamlines (black curves). Only panels (b) and (c) include streamlines in the interstellar medium. In panel (a) the view is looking directly at the sub-stagnation point on the terminal shock, while in (b) and (c) the stagnation point is to the right. 'The streamlines diverge from the poles as the anisotropic boundary condition on the shock (high-speed streams at the poles) evolve into the uniform flow of the interstellar wind in the downstream heliotail.

reported the magnetic field line topology and solar cycle imprint for a kinematic magnetic field in the heliosphere [ Nerney, Suess, and Schmahl, 1995]. A nonspherical shock would change the detailed shapes of both the magnetic field lines and the solar cycle imprint shown in that calculation. However, the largest difference shows up in using a free-slip versus a non-slip (our condition) boundary condition on the heliopause. The consequence of a free-slip boundary condition is to increase the flow speed down the heliotail, with a corresponding decrease in the cross-sectional area of the tail and in the thickness of the heliosheath on the upstream side of the heliosphere.

To the extent that velocity shear exists in the heliosheath inside the boundary layer at the heliopause, higher speed flow of the shocked solar wind in the interior of the heliosheath might lead to the importance of compressibility, which we have not included. We estimated the effects of compressibility in our model at about $15 \%$ (SN1), but we cannot be sure of the global effect with a non-slip boundary condition on the heliopause until a non-linear model is generated

Acknowledgments. This research was supported by the Cosmic and Heliospheric Physics Branch and by the Ulysses Project of NASA.

\section{References}

Kumar, S., and A. L. Broadfoot, Signatures of solar wind latitudinal structure in interplanetary Lyman- $\alpha$ emis- sions: Mariner 10 observations, Astrophys. J., 228, 302$311,1979$.

Lallement, R., J. I. Bertaux, and V. G. Kurt, Solar wind decrease at high heliographic latitudes detected from Prognoz interplanetary Lyman- $\alpha$ mapping, J. Geophys. Res., $90,1413-1423,1985$

Nerney, Steven, S. 'T. Suess, and E. J. Schmahl, Flow downstream of the heliospheric termination shock: 'The magnetic field on the heliopause, J. Geophys. Res., 98, 15,169$15,176,1993$.

Nerney, Steven, S. T. Suess, and E. J. Schmahl, Flow downstream of the heliospheric termination shock: Magnetic field line topology and solar cycle imprint, J. Geophys. Res., 100, A3, 3463-3471, 1995.

Parker, F.N., Interplanetary Dynamical Processes, Interscience, New York, 1963.

Steinolfson, R. S., Termination shock response to large-scale solar wind fluctuations, J. Geophys. Res., 99, 13,307$13,314,1994$.

Suess, S. T., The heliopause, Rev. Geophys., 28, 97-115, 1990

Suess, S.T. and S.F. Nerney (SN1), Flow downstream of the heliospheric termination shock, 1, Irrotational flow, J. Geophys. Res., 95, 6403-6412, 1990.

(SN2), Correction to "Flow downstream of the heliospheric terminal shock, 1, Irrotational flow", J. Geophys. Res., 96, 1883, 1991.

, The polar heliospheric magnetic field, Geophys. Res. Lett., 20, 329-332, 1993.

Space Science Lab/ES82, NASA Marshall Space Flight Center, Huntsville, Alabama 35812.

(received November, 1994; revised April, 1995; accepted May, 1995.) 